\title{
LES ALIMENTS COMPOSÉS MÉLASSÉS OU NON ET LA QUALITÉ DES FROMAGES (GRUYĖRE ET CARRÉ DE L'EST) (1)
}

par

\author{
F. FAVIN, R. FERRANDO, G. MOCQUOT \\ et G. THIEULIN
}

De très nombreux travaux semblent montrer que les aliments distribués aux vaches laitières pouvạient exercer une influence sur la qualité du fromage. Dans le cas du Gruyère, on redoute en particulier, le gonflement dû aux ferments butyriques, souvent observé lorsque les animaux reçoivent une ration renfermant un grand nombre de Clostridia. Ce type d'accident est observé notamment lorsque les animaux reçoivent de l'ensilage.

\section{Protocole expérimental}

Nous avons voulu, au cours de deux séries d'expériences, étudier un aspect de ce problème en examinant l'influence d'une distribution d'aliments composés et d'aliments mélassés aux vaches laitières.

Un premier essai eut lieu.dans la région de Reims, à la Ferme des Anglais. Nous tenons à remercier M. LAFFIte de nous avoir donné toutes facilités pour cette expérience préparatoire essentielle.

$\mathrm{Au}$ cours de celle-ci, 11 vaches laitières de race Frisonne française, réparties en deux lots de 6 et 5 sujets, reçurent de juin à octobre, alternativement chaque mois en opposant un lot à l'autre, un régime avec ou sans mélasse (2). L'analyse bactériologique du lait, pratiquée chaque semaine, a porté sur la flore totale, les coliformes, les levures, les moisissures et la présence éventuelle d'anaérobies thermo-résistants. L'ensemble des examens pratiqués ne devait pas montrer de différences entre les deux lots, notamment en ce qui concerne la teneur en anaérobies thermo-résistants. L'adjonction de mélasse à la ration n'eut aucune action sur la production laitière mais devait se répercuter favorablement sur le prix de revient de l'aliment (3).

Ces résultats nous encouragèrent à entreprendre dans la région de Poligny un deuxième groupe d'expériences portant sur un plus grand nombre de vaches laitières (environ 170) réparties en plu-

(1) C. R. Acad. Agr., 1959, no 6, 232.

(2) Ration contenant $25 \%$ de mélasse de betteraves.

(3) Ces expériences furent surveillées par un de nos assistants, M. Théodosiadés. 
sieurs étables (28). Certaines d'entre elles reçurent les aliments dont nous donnons ci-après les formules. La quantité de lait tránsformée en fromage était en moyenne (du $1^{\text {er }}$ novembre $1957 \mathrm{au}$ 1 er avril 1958) de 1.200 litres par jour.

Formule 1 Formule 2

\begin{tabular}{|c|c|c|}
\hline Orge & - & 20 \\
\hline$\ldots \ldots \ldots \ldots \ldots \ldots$ & 25 & - \\
\hline 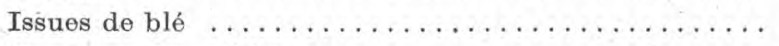 & 23 & 38 \\
\hline Tourteau d'arachide $\ldots \ldots \ldots \ldots \ldots \ldots \ldots$ & 25 & 15 \\
\hline 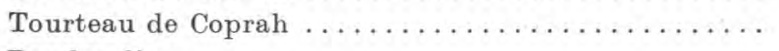 & 20 & 20 \\
\hline 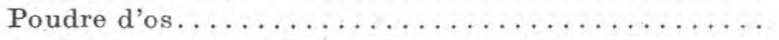 & 3,5 & 3,5 \\
\hline Carbonate de chaux $\ldots \ldots \ldots \ldots \ldots \ldots \ldots \ldots \ldots \ldots$ & 2 & 2 \\
\hline Chlorure de sodium & 1,5 & 1,5 \\
\hline
\end{tabular}

Les lots étaient ainsi répartis :

$1^{\circ}$ Un producteur de Vaux-sur-Poligny livrant son lait à l'Ecole d'Industrie laitière de Poligny a nourri ses vaches de la façon suivante :

$1^{\text {re }}$ période. 31 octobre au 30 novembre 1957 (matin) : Formule 2.

$2^{\mathrm{e}}$ période. 30 novembre (soir) au 31 décembre 1957 (matin) : Formule 1 (mélasse).

$3^{\text {e }}$ période. 31 décembre (soir) au 31 janvier 1958 : Formule 2.

Le lait servait à la fabrication de fromages type Carré de l'Est, dits " Pénitents ».

$2^{\circ}$ Par ailleurs, des étables livrant leur lait à la Fruitière de Buvilly furent réparties en deux groupes. Le premier, ou groupe témoin $\mathrm{T}$, comprend des étables dans lesquelles les vaches laitières reçoivent l'alimentation normalement distribuée à la ferme sans apport extérieur. Le lait livré par ce groupe de référence sert à fabriquer chaque jour un fromage de Comté, dit témoin T.

Le second, ou groupe d'expérience 3 , comprend 75 vaches réparties en 5 étables. Le rythme d'alimentation de ces étables fut le suivant :

$1^{\text {re }}$ période. Du $1^{\text {er }}$ au 30 novembre 1957 : alimentation habituelle sans apports extérieurs.

$2^{\mathrm{e}}$ période. Du $1^{\mathrm{er}}$ décembre 1957 au 31 janvier 1958 : alimentation avec la formule 1 (mélasse).

$3^{\mathrm{e}}$ période. Du 1 er février 1958 au 28 février 1958 : alimentation avec la formule 2 (sans mélasse). 
$4^{\mathrm{e}}$ période. Du $1^{\mathrm{er}}$ au 31 mars 1958 : alimentation habituelle sans apports extérieurs.

Tout le lait produit par l'ensemble des étables formant ce deuxième groupe sert à fabriquer chaque jour un fromage de Comté dit d'expérience E.

Tout au long de ces expériences, on analysa régulièrement les divers aliments des animaux. Les fourrages étaient dans l'ensemble récoltés trop tardivement. Leur teneur en matières protéiques était en moyenne située entre 8,5 et $9,5 \%$ et leur taux de phosphore était d'environ $0,19 \%$. Cependant, par suite d'un excès de calcium $(0,9$ à $1,3 \%)$, le rapport $\frac{\mathrm{Ca}}{\mathrm{p}}$ est très déséquilibré et voisin de 6 .

Sur le lait prélevé quatre à cinq fois par semaine dans les cuves de fabrication, on a déterminé le taux butyreux, celui des substances azotées et la teneur en calcium. En même temps, toute une série d'examens bactériologiques furent poursuivis; enfin, les fromages furent jugés en cave sur prélèvement à la sonde et, pour environ la moitié d'entre eux pris au hasard, sur une coupe que l'on a photographiée.

Ces expériences seront ultérieurement rapportées en détail. Nous en résumons ci-dessous les résultats principaux.

\section{Résultats}

Dans le cadre de la première expérience poursuivie avec le lait provenant de Vaux-sur-Poligny et destiné à la fabrication des fromages type "Carré de l'Est " aucun changement appréciable ne fut observé, tant dans la composition du lait que dans la qualité des fromages de chaque lot, celle-ci étant jugée en moyenne très bonne.

Nous avons résumé dans le tableau I les résultats concernant la deuxième expérience se rapportant à la production du fromage de Comté. Chacun des lots comprend autant de fromages qu'il y a de jours dans le mois.

Ces résultats confirment ceux obtenus dans l'expérience préparatoire. Ils montrent que la distribution des différents régimes ci-dessus mentionnés n'a pas exercé d'influence décelable sur la qualité des fromages dans lés conditions de notre expérimentation, c'est-à-dire avec des aliments de bonne qualité bactériologique. Les variations de qualité observées au cours de celle-ci sont vraisemblablement dues à d'autres causes que la distribution ou non distribution. d'aliments composés mélassés ou non. A cet égard, il convient de noter qu'en l'absence de tout apport d'aliment com- 
TABLEAU I

NOTE DE QUALITÉ EXPRIMÉE DE 0 A 20, ATTRIBUÉ A CHAGUN DES LOTS DE FROMAGE T OU D'EXPÉRIENGE E ET AUX DEUX LOTS

$\frac{\mathbf{E}+\mathbf{T}}{\mathbf{2}}$

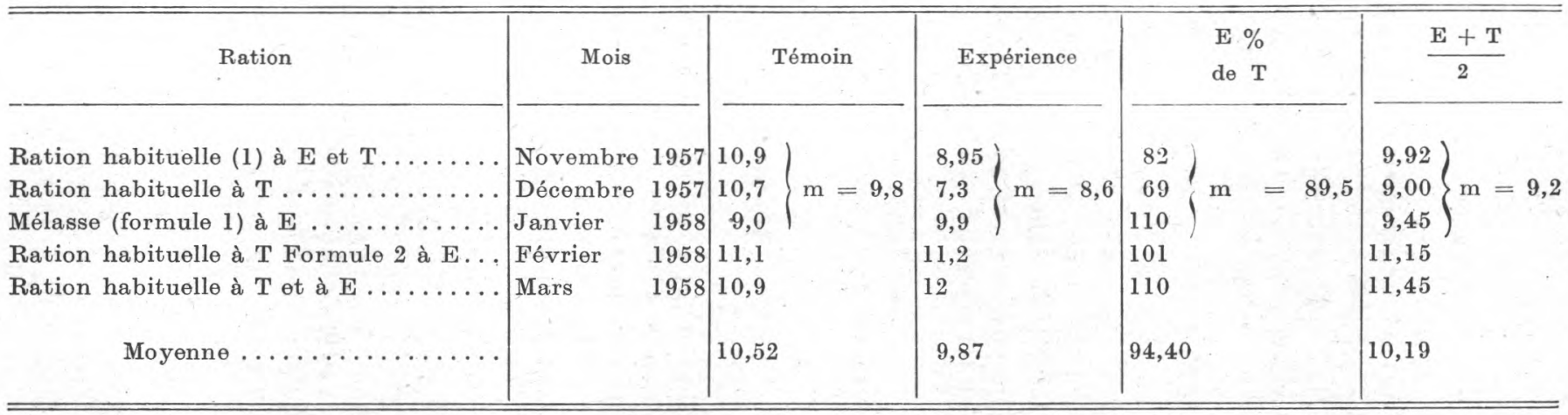

(1) Ration sans apport d'aliments extérieurs. 
posé, la qualité bactériologique du lait produit par le lot $\mathrm{E}$ fut moins bonne en novembre que celle du lot $\mathrm{T}$.

On sait aussi que la qualité d'un fromage dépend beaucoup de la technique mise en ouvre par le fromager. Un changement de fromager, intervenu le $1^{\text {er }}$ janvier 1958 , a pu exercer à cet égard une certaine influence. On a noté par la suite, en février et mars, une amélioration de la qualité des fromages. L'expérience que deux d'entre nous (F. F. et G. M.) possèdent de nombreux essais antérieurs montre qu'il est difficile, dans les conditions pratiques du travail en fruitière, d'éviter les causes de variations et d'obtenir une fabrication possédant une qualité constante.

Au point de vue de la production laitière, les différences entre chaque période expérimentale sont nulles et le type d'alimentation n'exerça pas d'influence appréciable sur la composition des laits.

En conclusion, les éventuelles répercussions dues à la distribution d'aliments composés mélassés ou non paraissent moins importantes que les causes de variations de la qualité des fromages s'exerçant habituellement dans un chalet de fromagerie du Jura ou que celles se faisant sentir au niveau de l'étable.

(Station de Recherches laitières et Ecole d'Industrie laitière de Poligny, Laboratoire d'Alimentation de l'Ecole Nationale Vétérinaire d'Alfort, Laboratoire du Service Vétérinaire de la Seine.)

\section{RECHERCHE DES ALGINATES DANS LES PRODUITS LAITIERS}

(Note de laboratoire)

$$
\text { par JEAN PIEN }
$$

La recherche des épaississants ou gélifiants organiques dans les denrées alimentaires en général et dans les produits laitiers en particulier présente de sérieuses difficultés, non seulement dans l'extraction et la purification de ces substances en présence d'un grand excès de matières protéiques, mais encore parce qu'on ne dispose guère de réactions spécifiques permettant l'identification de ces substances.

Au cours de ces dernières années, cependant, a été proposée la mise en évidence globale de tous les épaississants organiques de structure glucidique par la réaction de l'anthrone (ou hydroxydihydro-anthracène, obtenu par réduction de l'anthraquinone).

(1) Annales Fals, et Fraudes, 1959, 52, 197. 